PROCEEDINGS OF THE

AMERICAN MATHEMATICAL SOCIETY

Volume 132, Number 11, Pages 3337-3344

S 0002-9939(04)07427-1

Article electronically published on May 21, 2004

\title{
THE FIRST DIRAC EIGENVALUES ON MANIFOLDS WITH POSITIVE SCALAR CURVATURE
}

\author{
CHRISTIAN BÄR AND MATTIAS DAHL \\ (Communicated by Józef Dodziuk)
}

\begin{abstract}
We show that on every compact spin manifold admitting a Riemannian metric of positive scalar curvature Friedrich's eigenvalue estimate for the Dirac operator can be made sharp up to an arbitrarily small given error by choosing the metric suitably.
\end{abstract}

\section{Introduction AND STATEMENT OF THE RESULT}

On an $n$-dimensional compact Riemannian spin manifold $M$ the Dirac operator has discrete real spectrum consisting only of eigenvalues of finite multiplicity. If the manifold has positive scalar curvature, then 0 lies in a spectral gap. More precisely, Friedrich [4] showed that all eigenvalues $\lambda$ of the Dirac operator satisfy

$$
\lambda^{2} \geq \frac{n}{4(n-1)} \min _{M} \text { Scal } .
$$

This inequality is sharp in the sense that there are examples in all dimensions where equality is attained for the eigenvalue of smallest modulus. The standard sphere provides such examples. Equality in this estimate implies strong restrictions on the manifold. The manifold must be Einstein and the corresponding eigenspinor then must be a Killing spinor. The geometric types of manifolds admitting such Killing spinors are classified; see [2]. In particular, in even dimension $n \neq 6$ only the standard sphere occurs.

The dimension-dependent coefficient $c_{n}=\frac{n}{4(n-1)}$ in the estimate can be improved if one imposes geometric assumptions on the metric. Kirchberg [6] 7] showed that for Kähler metrics $c_{n}$ can replaced by $\frac{n+2}{4 n}$ if the complex dimension $\frac{n}{2}$ is odd, and by $\frac{n}{4(n-2)}$ if $\frac{n}{2}$ is even. For quaternionic Kähler manifolds Kramer, Weingart, and Semmelmann [8] showed that $c_{n}$ can be improved to $\frac{n+12}{4(n+8)}$.

Alexandrov, Grantcharov, and Ivanov [1] showed that if there exists a parallel one-form on $M$, then $c_{n}$ can be replaced by $c_{n-1}=\frac{n-1}{4(n-2)}$. In a recent paper Moroianu and Ornea [9] weakened the assumption on the 1-form from parallel to

Received by the editors July 2, 2003.

2000 Mathematics Subject Classification. Primary 53C27.

Key words and phrases. Dirac operator, eigenvalue, positive scalar curvature, Friedrich's estimate.

The first author has been partially supported by the Research and Training Networks HPRNCT-2000-00101 "EDGE" and HPRN-CT-1999-00118 "Geometric Analysis" funded by the European Commission.

(C)2004 American Mathematical Society 
harmonic with constant length. They believed that the condition of having constant length is also unnecessary and conjectured [9, Conj. 1]: All Dirac eigenvalues $\lambda$ on an $n$-dimensional compact Riemannian spin manifold with nonzero first Betti number satisfy

$$
\lambda^{2} \geq \frac{n-1}{4(n-2)} \min _{M} \text { Scal } .
$$

We show here that this conjecture is false. If $D_{M}$ is the Dirac operator on a closed Riemannian spin manifold $M$, then the spectrum of $D_{M}^{2}$ is of the form

$$
0 \leq \lambda_{1}\left(D_{M}^{2}\right) \leq \lambda_{2}\left(D_{M}^{2}\right) \leq \lambda_{3}\left(D_{M}^{2}\right) \leq \cdots \rightarrow \infty
$$

with each eigenvalue repeated according to its multiplicity. We prove

Theorem 1. Let $M$ be a compact n-dimensional Riemannian spin manifold with positive scalar curvature.

Then there exists a smooth one-parameter family of Riemannian metrics $g_{\varepsilon}$ on $M, \varepsilon \in\left(0, \varepsilon_{0}\right]$, such that

- $\mathrm{Scal}_{g_{\varepsilon}} \geq n(n-1)$, and

- $\frac{n^{2}}{4} \leq \lambda_{1}\left(D_{M, g_{\varepsilon}}^{2}\right) \leq \cdots \leq \lambda_{2^{[n / 2]+1}}\left(D_{M, g_{\varepsilon}}^{2}\right) \leq \frac{n^{2}}{4}+\varepsilon$.

Here $[n / 2]$ denotes the integer part of $n / 2$. The lower eigenvalue bound is nothing but Friedrich's estimate. Thus the theorem says in particular that Friedrich's estimate can always be made "almost sharp" by choosing suitable metrics. Hence the dimension-dependent coefficient $\frac{n}{4(n-1)}$ in the estimate cannot be improved by imposing additional topological assumptions like bounds on the Betti numbers.

This is indeed remarkable, since equality in Friedrich's estimate is so restrictive on the manifold. The manifold then has to be Einstein with positive Einstein constant. So it has positive Ricci curvature, and hence its fundamental group is finite by the Bonnet-Myers theorem. In particular, the first Betti number must vanish. On the other hand, our theorem says that "almost equality" in Friedrich's estimate does not impose any topological restriction on the manifold.

\section{THE PROOF}

We start by proving two preliminary lemmas.

Lemma 2. Let $M$ be a compact Riemannian spin manifold of dimension $n \geq 3$, and let $p \in M$.

Then for each $k \in \mathbb{N}$ and each $\delta>0$ there exists $R_{k}(\delta)>0$ such that for each compact $n$-dimensional Riemannian spin manifold $\widetilde{M}$ containing an isometric copy of $M-\bar{B}_{r}(p)$ as an open subset (with the same restricted spin structure), where $B_{r}(p)$ is the geodesic ball about $p$ of radius $r, 0<r \leq R_{k}(\delta)$, we have

$$
\lambda_{j}\left(D_{\widetilde{M}}^{2}\right) \leq \lambda_{j}\left(D_{M}^{2}\right)+\delta
$$

for all $j=1, \ldots, k$. Moreover, $R_{k}$ can be chosen so that it depends smoothly on $\delta$.

Proof. Denote by $\mathcal{H}$ the $j$-dimensional vector space spanned by the eigenspinors corresponding to the eigenvalues $\lambda_{1}\left(D_{M}^{2}\right), \ldots, \lambda_{j}\left(D_{M}^{2}\right)$ of $D_{M}^{2}$.

Fix $r_{0}>0$ such that $2 r_{0}<\operatorname{injrad}(p)$, the injectivity radius of $p$. For $0<r \leq r_{0}$ choose a smooth cutoff function $\chi \in C^{\infty}(M)$ satisfying

- $0 \leq \chi \leq 1$ on all of $M$,

- $\chi \equiv 0$ on $B_{r}(p)$, 
- $\chi \equiv 1$ on $M-B_{2 r}(p)$, and

- $|\operatorname{grad} \chi| \leq 2 / r$ on $M$.

By the unique continuation property of eigenspinors the space $\widetilde{\mathcal{H}}:=\{\chi \cdot \varphi: \varphi \in$ $\mathcal{H}\}$ is also $j$-dimensional. Since all $\chi \varphi \in \widetilde{\mathcal{H}}$ vanish on $\bar{B}_{r}(p)$ we may also consider them as spinors on $\widetilde{M}$.

There is a constant $c_{1}$ such that $\operatorname{vol}\left(B_{2 r}(p)\right) \leq c_{1} r^{n}$. Since $\mathcal{H}$ is finite dimensional, there exists $c_{2}$ such that $\|\varphi\|_{L^{\infty}(M)}^{2} \leq c_{2}\|\varphi\|_{L^{2}(M)}^{2}$ for all $\varphi \in \mathcal{H}$. Fix $\alpha>0$. For any $\chi \varphi \in \widetilde{\mathcal{H}}$ we compute

$$
\begin{aligned}
\int_{\widetilde{M}}\left|D_{\widetilde{M}}(\chi \varphi)\right|^{2} d V & =\int_{M}\left|D_{M}(\chi \varphi)\right|^{2} d V \\
& =\int_{M}\left|\chi D_{M} \varphi+\operatorname{grad} \chi \cdot \varphi\right|^{2} d V \\
& \leq \int_{M}\left((1+\alpha) \chi^{2}\left|D_{M} \varphi\right|^{2}+\left(1+\frac{1}{\alpha}\right)|\operatorname{grad} \chi|^{2}|\varphi|^{2}\right) d V \\
& \leq(1+\alpha) \int_{M}\left|D_{M} \varphi\right|^{2} d V+\left(1+\frac{1}{\alpha}\right) \int_{B_{2 r}(p)} \frac{4}{r^{2}}|\varphi|^{2} d V \\
& \leq(1+\alpha) \lambda_{j}\left(D_{M}^{2}\right)\|\varphi\|_{L^{2}(M)}^{2}+\left(1+\frac{1}{\alpha}\right) \frac{4}{r^{2}} \operatorname{vol} B_{2 r}(p)\|\varphi\|_{L^{\infty}(M)}^{2} \\
& \leq\left((1+\alpha) \lambda_{j}\left(D_{M}^{2}\right)+\left(1+\frac{1}{\alpha}\right) 4 c_{1} r^{n-2} c_{2}\right)\|\varphi\|_{L^{2}(M)}^{2}
\end{aligned}
$$

Moreover,

$$
\begin{aligned}
\int_{\widetilde{M}}|\chi \varphi|^{2} d V & =\int_{M}|\chi \varphi|^{2} d V \\
& \geq \int_{M-B_{2 r}(p)}|\varphi|^{2} d V \\
& \geq \int_{M}|\varphi|^{2} d V-\|\varphi\|_{L^{\infty}(M)}^{2} \operatorname{vol}\left(B_{2 r}(p)\right) \\
& \geq\left(1-c_{1} c_{2} r^{n}\right)\|\varphi\|_{L^{2}(M)}^{2} .
\end{aligned}
$$

Thus for all $\chi \varphi \in \widetilde{\mathcal{H}}$ the Rayleigh quotient of $D_{\widetilde{M}}^{2}$ and hence $\lambda_{j}\left(D_{\widetilde{M}}^{2}\right)$ are bounded from above by

$$
\frac{\int_{\widetilde{M}}\left|D_{\widetilde{M}}(\chi \varphi)\right|^{2} d V}{\int_{\widetilde{M}}|\chi \varphi|^{2} d V} \leq \frac{(1+\alpha) \lambda_{j}\left(D_{M}^{2}\right)+4 c_{1} c_{2}\left(1+\frac{1}{\alpha}\right) r^{n-2}}{1-c_{1} c_{2} r^{n}}
$$

The lemma follows by first choosing $\alpha$ so small that $\alpha \lambda_{j}\left(D_{M}^{2}\right)<\frac{\delta}{2}$ and then choosing $r$ sufficiently small.

Lemma 3. Let $p \in S^{n}, n \geq 2$. Let $S>0$.

There exists a smooth one-parameter family of Riemannian metrics $h_{\delta}$ on $S^{n}$, $\delta \in\left(0, \delta_{0}\right]$, such that

- $\operatorname{Scal}_{h_{\delta}} \geq n(n-1)$,

- $\left.\mathrm{Scal}_{h_{\delta}}\right|_{B_{\widehat{R}(\delta)}(p)} \geq S$, where $\widehat{R}(\delta)>0$ depends smoothly on $\delta$, and

- $\lambda_{2^{[n / 2]+1}}\left(D_{h_{\delta}}^{2}\right) \leq \frac{n^{2}}{4}+\delta$. 


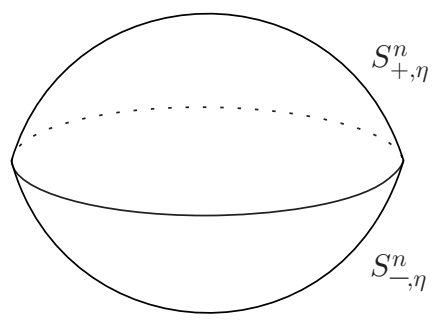

FIGURE 1.

Proof. The idea of the proof is this. Consider the sphere with its standard metric embedded in Euclidean space $\mathbb{R}^{n+1}, S^{n}=\left\{\left(t, x_{1}, \ldots, x_{n}\right) \in \mathbb{R}^{n+1}:|(t, x)|^{2}=1\right\}$. Call $S_{+}^{n}:=\left\{\left(t, x_{1}, \ldots, x_{n}\right) \in \mathbb{R}^{n+1}:|(t, x)|^{2}=1, t \geq 0\right\}$ the northern hemisphere and $S_{-}^{n}:=\left\{\left(t, x_{1}, \ldots, x_{n}\right) \in \mathbb{R}^{n+1}:|(t, x)|^{2}=1, t \leq 0\right\}$ the southern hemisphere. Move the northern hemisphere by a small amount $\eta>0$ to the south (i.e., in direction $\left.-e_{0}=(-1,0, \ldots, 0)\right)$ and cut off everything that gets moved south of the hyperplane $\{0\} \times \mathbb{R}^{n}$. This yields the northern cup $S_{+, \eta}^{n}:=\left\{(t, x) \in \mathbb{R}^{n+1}\right.$ : $\left.|(t+\eta, x)|^{2}=1, t \geq-\eta\right\}$. Similarly, move the southern hemisphere to the north by the amount $\eta$, cut off everything north of the equatorial hyperplane, and obtain the southern cup $S_{-, \eta}^{n}:=\left\{(t, x) \in \mathbb{R}^{n+1}:|(t-\eta, x)|^{2}=1, t \leq \eta\right\}$. The union $S_{+, \eta}^{n} \cup S_{-, \eta}^{n}$ is a hypersurface in $\mathbb{R}^{n+1}$, singular along the equator $\partial S_{+, \eta}^{n}=\partial S_{-, \eta}^{n}=S_{+, \eta}^{n} \cap S_{-, \eta}^{n}$, smooth elsewhere with constant sectional curvature $K \equiv 1$ and constant mean curvature $H \equiv 1$.

As we will see, careful smoothing of this hypersurface in a neighborhood of the equator yields a hypersurface $S_{\eta}^{n}$ diffeomorphic to $S^{n}$ such that

- Scal $\geq n(n-1)$ everywhere,

- Scal $\geq S$ in the $\eta^{2}\left(1-4 \eta^{2}\right)$-tubular neighborhood of the equator, and

- $\frac{1}{\operatorname{vol}\left(S_{\eta}^{n}\right)} \int_{S_{\eta}^{n}} H^{2} d V \leq 1+\mathrm{O}(\eta)$.

If $p$ was placed on the equator in the beginning, which we may assume, then the statements on the scalar curvature follow immediately. The bound on the Dirac eigenvalue is a consequence of

$$
\frac{\lambda_{1}\left(D_{M}^{2}\right)+\lambda_{2^{[n / 2]+1}}\left(D_{M}^{2}\right)}{2} \leq \frac{n^{2}}{4 \operatorname{vol}(M)} \int_{M} H^{2} d V
$$

for any compact oriented hypersurface $M$ of $\mathbb{R}^{n+1}$; see [3, Thm. 4.7]. Namely, by Friedrich's inequality we have

$$
\begin{aligned}
\lambda_{2^{[n / 2]+1}}\left(D_{S_{\eta}^{n}}^{2}\right) & \leq 2 \frac{n^{2}}{4 \operatorname{vol}\left(S_{\eta}^{n}\right)} \int_{S_{\eta}^{n}} H^{2} d V-\lambda_{1}\left(D_{S_{\eta}^{n}}^{2}\right) \\
& \leq \frac{n^{2}}{2}(1+\mathrm{O}(\eta))-\frac{n}{n-1} \frac{n(n-1)}{4} \\
& =\frac{n^{2}}{4}(1+\mathrm{O}(\eta)) .
\end{aligned}
$$




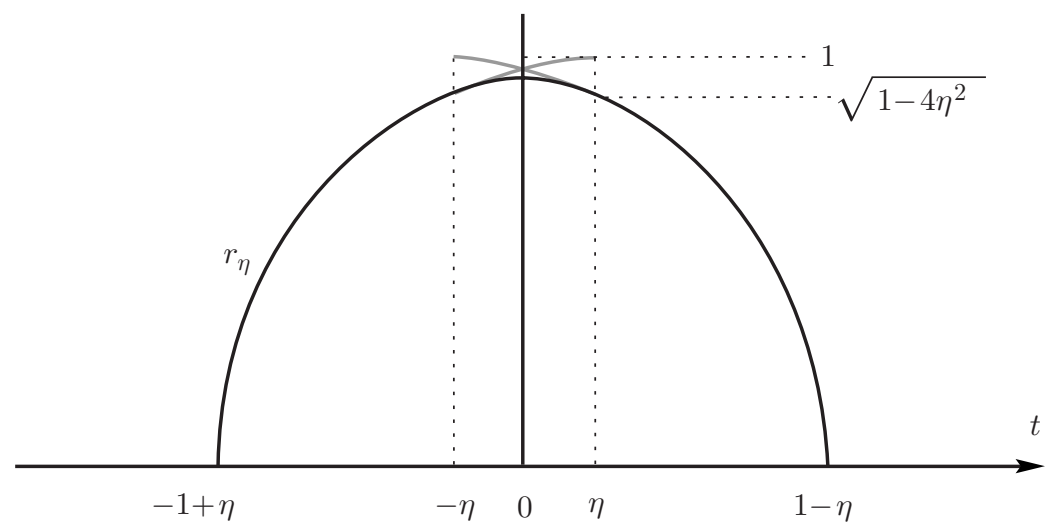

FiguRE 2. Graph of $r_{\eta}$

To make the smoothing of $S_{+, \eta}^{n} \cup S_{-, \eta}^{n}$ explicit we start with the case of dimension $n=2$. The 2 -sphere is a surface of revolution, parametrized by

$$
F:[-1,1] \times S^{1} \rightarrow \mathbb{R}^{3}, \quad F(t, \theta)=(t, r(t) \cos (\theta), r(t) \sin (\theta))
$$

with $r(t)=\sqrt{1-t^{2}}$. The general formula for the principal curvatures of a surface of revolution is

$$
\kappa_{t}=\frac{-\ddot{r}}{\left(1+\dot{r}^{2}\right)^{3 / 2}}
$$

and

$$
\kappa_{\theta}=\frac{1}{r \sqrt{1+\dot{r}^{2}}}
$$

In our case of the sphere, $\kappa_{t}=\kappa_{\theta}=1$. The smoothed hypersurface $S_{\eta}^{2}$ will also be a surface of revolution with radius function $r_{\eta}:[-1+\eta, 1-\eta] \rightarrow \mathbb{R}$ chosen subject to the following conditions:

(1) $r_{\eta}$ is smooth on $(-1+\eta, 1-\eta)$ and even,

(2) $r_{\eta}(t)=\sqrt{1-(t-\eta)^{2}}$ for $t \in[-1+\eta,-\eta]$,

(3) $r_{\eta}(t)=\sqrt{1-(t+\eta)^{2}}$ for $t \in[\eta, 1-\eta]$,

(4) $\sqrt{1-4 \eta^{2}} \leq r_{\eta}(t) \leq \frac{1}{\sqrt{1-4 \eta^{2}}}$ for $t \in[-\eta, \eta]$,

(5) $\left|\dot{r}_{\eta}(t)\right| \leq \frac{2 \eta}{\sqrt{1-4 \eta^{2}}}$ for $t \in[-\eta, \eta]$,

(6) $\ddot{r}_{\eta}<0$ on $(-1+\eta, 1-\eta)$,

(7) $\ddot{r}_{\eta}(t)=-2 S$ for $t \in\left[-\eta^{2}, \eta^{2}\right]$, and

(8) $-2 S \leq \ddot{r}_{\eta}(t) \leq \frac{-1}{\left(1-4 \eta^{2}\right)^{3 / 2}}$ for $t \in[-\eta, \eta]$.

For (2) and (3) to make sense we assume that $\eta<\frac{1}{2}$. By (2) we have $\dot{r}_{\eta}(-\eta)=$ $\frac{2 \eta}{\sqrt{1-4 \eta^{2}}}$, and by (3) we get $\dot{r}_{\eta}(\eta)=\frac{-2 \eta}{\sqrt{1-4 \eta^{2}}}$. Thus (6) implies (5).

Moreover, by (2) and (3) we obtain $r_{\eta}( \pm \eta)=\sqrt{1-4 \eta^{2}}$ and so, by (6), we get $r_{\eta} \geq \sqrt{1-4 \eta^{2}}$ on $[-\eta, \eta]$. By (5) we see that $r_{\eta}(t) \leq r_{\eta}(-\eta)+\eta \frac{2 \eta}{\sqrt{1-4 \eta^{2}}} \leq \frac{1}{\sqrt{1-4 \eta^{2}}}$ for $t \in[-\eta, 0]$ and similarly for $t \in[0, \eta]$. Hence (4) is also a consequence of (2), $(3)$, and (6).

Condition (6) follows from (2), (3), and (8). 


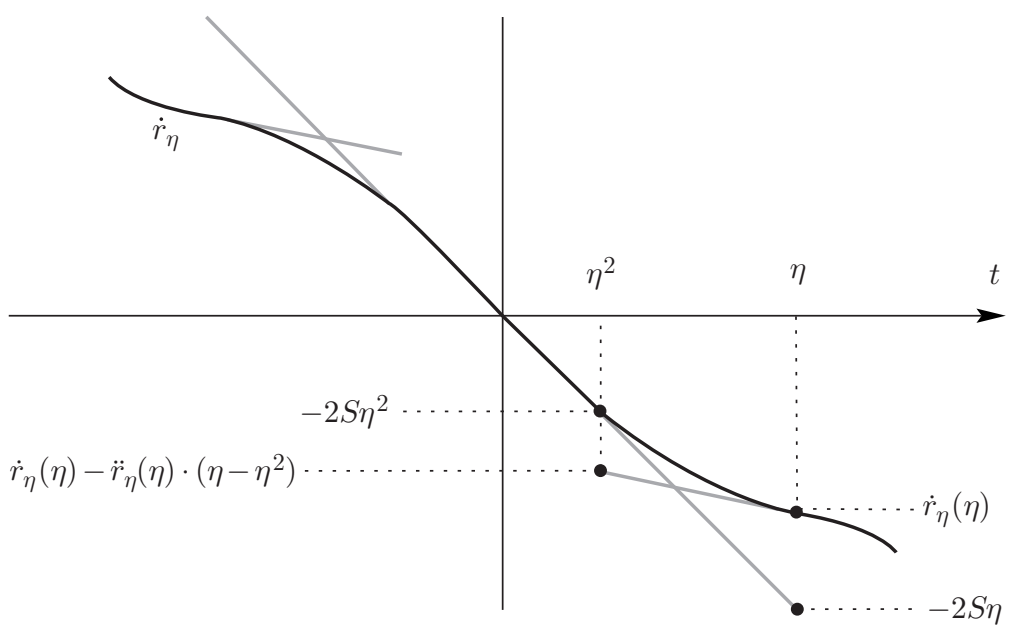

FiguRE 3. Graph of $\dot{r}_{\eta}$

To make sure that conditions (2), (3), (7), and (8) can be realized we need to assume $S>1$, which we do without loss of generality, and that $\eta$ is so small that

(a) $2 S \eta^{2}<-\dot{r}_{\eta}(\eta)=\frac{2 \eta}{\sqrt{1-4 \eta^{2}}}$,

(b) $-2 S \eta<\dot{r}_{\eta}(\eta)$, i.e., $S>\frac{1}{\sqrt{1-4 \eta^{2}}}$, and

(c) $-2 S \eta^{2}>\dot{r}_{\eta}(\eta)-\ddot{r}_{\eta}(\eta) \cdot\left(\eta-\eta^{2}\right)$, i.e., $S<\frac{\frac{1}{\eta}+1-8 \eta}{2\left(1-4 \eta^{2}\right)^{3 / 2}}$.

Conditions (1)-(8) together with the explicit formulas for the principal curvatures imply

$$
\begin{array}{cl}
1 \leq \kappa_{t}(t, \theta) \leq 2 S & \text { for } t \in[-\eta, \eta], \\
\kappa_{t}(t, \theta) \geq 2 S\left(1-4 \eta^{2}\right)^{3 / 2} & \text { for } t \in\left[-\eta^{2}, \eta^{2}\right], \\
1-4 \eta^{2} \leq \kappa_{\theta}(t, \theta) \leq \frac{1}{\sqrt{1-4 \eta^{2}}} & \text { for } t \in[-\eta, \eta] .
\end{array}
$$

At this point we return to the general case of dimension $n \geq 2$. If $n \geq 3$, then we perform exactly the same smoothing, i.e., we use the same warping function $r_{\eta}(t)$, where $t$ is the first Cartesian coordinate in $\mathbb{R}^{n+1}$. For every 3-dimensional vector subspace $E \subset \mathbb{R}^{n+1}$ containing $e_{0}$, the reflection across $E$ is an isometry of $\mathbb{R}^{n+1}$ leaving the smoothed hypersurface $S_{\eta}^{n}$ invariant. Hence the fixed point set $E \cap S_{\eta}^{n}$ is a totally geodesic submanifold of $S_{\eta}^{n}$, so that its principal curvatures are also principal curvatures of $S_{\eta}^{n}$. Therefore the principal curvatures $\kappa_{1}, \ldots, \kappa_{n}$ of $S_{\eta}^{n}$ satisfy

$$
\begin{array}{cl}
1 \leq \kappa_{1}(t, \theta) \leq 2 S & \text { for } t \in[-\eta, \eta], \\
\kappa_{1}(t, \theta) \geq 2 S\left(1-4 \eta^{2}\right)^{3 / 2} & \text { for } t \in\left[-\eta^{2}, \eta^{2}\right], \\
1-4 \eta^{2} \leq \kappa_{j}(t, \theta) \leq \frac{1}{\sqrt{1-4 \eta^{2}}} & \text { for } t \in[-\eta, \eta], j=2, \ldots, n .
\end{array}
$$


This implies, for the scalar curvature Scal $=\sum_{i \neq j} \kappa_{i} \kappa_{j}$ and the mean curvature $H=\frac{1}{n} \sum_{j} \kappa_{j}$,

$$
\begin{array}{cl}
\text { Scal } \geq n(n-1)\left(1-4 \eta^{2}\right)^{2} & \text { for } t \in[-\eta, \eta], \\
\text { Scal }>S & \text { for } t \in\left[-\eta^{2}, \eta^{2}\right], \\
H \leq 2 S & \text { for } t \in[-\eta, \eta], \\
H=1 & \text { for } t \in[-1+\eta,-\eta] \cup[\eta, 1-\eta], \\
\text { Scal }=n(n-1) & \text { for } t \in[-1+\eta,-\eta] \cup[\eta, 1-\eta] .
\end{array}
$$

The volume of the part of $S_{\eta}^{n}$ contained in the strip $-\eta \leq t \leq \eta$ is of order $\mathrm{O}(\eta)$. Thus

$$
\begin{aligned}
\frac{1}{\operatorname{vol} S_{\eta}^{n}} \int_{S_{\eta}^{n}} H^{2} d V & =1+\frac{1}{\operatorname{vol} S_{\eta}^{n}} \int_{S_{\eta}^{n}}\left(H^{2}-1\right) d V \\
& \leq 1+\frac{1}{\operatorname{vol} S_{\eta}^{n}} \int_{S_{\eta}^{n} \cap\{-\eta \leq t \leq \eta\}} 2 S d V \\
& =1+\mathrm{O}(\eta) .
\end{aligned}
$$

Hence $S_{\eta}^{n}$ satisfies

- Scal $\geq n(n-1)\left(1-4 \eta^{2}\right)^{2}$ everywhere,

- Scal $\geq S$ in the $\eta^{2}$-tubular neighborhood of the equator, and

- $\frac{1}{\operatorname{vol}\left(S_{\eta}^{n}\right)} \int_{S_{\eta}^{n}} H^{2} d V \leq 1+\mathrm{O}(\eta)$.

Multiplying $S_{\eta}^{n}$ by the factor $\left(1-4 \eta^{2}\right)$ yields

- Scal $\geq n(n-1)$ everywhere,

- Scal $\geq \frac{S}{\left(1-4 \eta^{2}\right)^{2}} \geq S$ in the $\eta^{2}\left(1-4 \eta^{2}\right)$-tubular neighborhood of the equator, and

- $\frac{1}{\operatorname{vol}\left(S_{\eta}^{n}\right)} \int_{S_{\eta}^{n}} H^{2} d V \leq \frac{1+\mathrm{O}(\eta)}{\left(1-4 \eta^{2}\right)^{2}}=1+\mathrm{O}(\eta)$.

Replacing a suitable multiple of $\eta$ by $\delta$ concludes the proof.

Proof of the Theorem. Without loss of generality we may assume that $M$ is connected. If $M$ is 2 -dimensional, it must be the 2-sphere. The constant family $g_{\varepsilon}=g_{\text {can }}$ does the job, where $g_{\text {can }}$ is the standard metric of constant Gauss curvature 1 , because

- Scal $_{g_{\text {can }}} \equiv 2=n(n-1)$, and

- $\lambda_{1}\left(D_{S^{2}, g_{\text {can }}}^{2}\right)=\cdots=\lambda_{4}\left(D_{S^{2}, g_{\text {can }}}^{2}\right)=1=\frac{n^{2}}{4}$.

From now on let $n \geq 3$. Pick a Riemannian metric $h$ on $M$ such that $\operatorname{Scal}_{h} \geq$ $2 n(n-1)$. Apply Lemma 3 with $S=2 n(n-1)$ and obtain a smooth one-parameter family of metrics $h_{\delta}$ on $S^{n}$ such that

- $\operatorname{Scal}_{h_{\delta}} \geq n(n-1)$,

- $\left.\operatorname{Scal}_{h_{\delta}}\right|_{B_{\widehat{R}(\delta)}(p)} \geq 2 n(n-1)$, and

- $\lambda_{2^{[n / 2]+1}}\left(D_{h_{\delta}}^{2}\right) \leq \frac{n^{2}}{4}+\delta$.

Let $r(\delta)$ be a smooth function of $\delta$ such that $0<r(\delta) \leq \min \left\{R_{2^{[n / 2]+1}}(\delta), \widehat{R}(\delta)\right\}$, where $R_{2^{[n / 2]+1}}(\delta)$ and $\widehat{R}(\delta)$ are as in Lemmas 2 and 3 Now form the connected sum $\widetilde{M}$ of $\left(S^{n}, h_{\delta}\right)$ and $(M, h)$ such that the metric $h_{\delta}$ remains unchanged outside $B_{r(\delta)}(p)$. Thus $\widetilde{M}$ contains an isometric copy of $S^{n}-\bar{B}_{r(\delta)}(p)$, and by Lemma 2 we obtain

$$
\lambda_{2^{[n / 2]+1}}\left(D_{\widetilde{M}}^{2}\right) \leq \lambda_{2^{[n / 2]+1}}\left(D_{S^{n}, h_{\delta}}^{2}\right)+\delta \leq \frac{n^{2}}{4}+2 \delta .
$$


Performing connected sums in dimension $n \geq 3$ (or, more generally, surgery in codimension $\leq 3$ ) does not decrease scalar curvature too much if the metric on the connected sum is chosen carefully; see [5, Proof of Theorem A] and [10, Proof of Theorem 3.1]. Since Scal $\geq 2 n(n-1)$ on $B_{r(\delta)}(p)$ as well as on $M$, we may assume that the metric on $\widetilde{M}$ still has scalar curvature Scal $\geq n(n-1)$. Moreover, the construction of the metric on $\widetilde{M}$ can be done smoothly in $\delta$. Since $\widetilde{M}$ is diffeomorphic to the original manifold $M$, the substitution $\varepsilon=2 \delta$ yields the theorem.

\section{REFERENCES}

[1] B. Alexandrov, G. Grantcharov, and S. Ivanov, An estimate for the first eigenvalue of the Dirac operator on compact riemannian spin manifold admitting parallel one-form, J. Geom. Phys. 28, (1998), 263-270. MR 99k:58182

[2] C. Bär, Real Killing spinors and holonomy, Commun. Math. Phys. 154 (1993), 509-521. MR 94i:53042

[3] $ـ$ Extrinsic bounds for eigenvalues of the Dirac operator, Ann. Glob. Anal. Geom. 16 (1998), 573-596. MR 99k:58183

[4] T. Friedrich, Der erste Eigenwert des Dirac-Operators einer kompakten Riemannschen Mannigfaltigkeit nicht-negativer Krümmung, Math. Nachr. 97 (1980), 117-146. MR 82g:58088

[5] M. Gromov and H. B. Lawson, The classification of simply connected manifolds of positive scalar curvature, Ann. Math., II. Ser. 111 (1980), 423-434. MR 81h:53036

[6] K.-D. Kirchberg, An estimation for the first eigenvalue of the Dirac operator on closed Kähler manifolds of positive scalar curvature, Ann. Global Anal. Geom. 4 (1986), 291-325. MR 89b:58221

[7] — The first eigenvalue of the Dirac operator on Kähler manifolds, J. Geom. Phys. 7 (1990), 449-468. MR 92h:58199

[8] W. Kramer, U. Semmelmann, and G. Weingart, Eigenvalue estimates for the Dirac operator on quaternionic Kähler manifolds, Math. Z. 230 (1999), 727-751. MR 2000d:58046

[9] A. Moroianu and L. Ornea, Eigenvalue estimates for the Dirac operator and harmonic 1forms of constant length, C. R. Acad. Sci. Paris Série I 338 (2004), 561-564.

[10] J. Rosenberg and S. Stolz, Metrics of positive scalar curvature and connections with surgery, Surveys on surgery theory, Vol. 2, Ann. of Math. Stud. 149, Princeton Univ. Press, 2001, 353-386. MR 2002f:53054

Institut für Mathematik, Universität Potsdam, PF 601553, 14415 Potsdam, Germany

E-mail address: baer@math.uni-potsdam.de

Institutionen för Matematik, Kungliga Tekniska Högskolan, 10044 Stockholm, SweDEN

E-mail address: dahl@math.kth.se 